\title{
Téoros
}

Revue de recherche en tourisme

\section{Le tourisme essentiel aux compagnies aériennes}

\section{Philippe Sureau}

Volume 6, numéro 1, mars 1987

Tourisme et transports

URI : https://id.erudit.org/iderudit/1080517ar

DOI : https://doi.org/10.7202/1080517ar

Aller au sommaire du numéro

Éditeur(s)

Université du Québec à Montréal

ISSN

0712-8657 (imprimé)

1923-2705 (numérique)

Découvrir la revue

Citer cet article

Sureau, P. (1987). Le tourisme essentiel aux compagnies aériennes. Téoros, 6(1),

4-6. https://doi.org/10.7202/1080517ar d'utilisation que vous pouvez consulter en ligne.

https://apropos.erudit.org/fr/usagers/politique-dutilisation/ 


\section{Le tourisme essentiel aux compagnies aériennes}

par Philippe Sureau*

Le consommateur vacancier qui pénètre dans une agence de voyages voit son attention immediatement sollicitée par les noms, les marques, les identifications de compagnies aériennes. Les brochures de voyages et les affiches sont ornées du logo des transporteurs aériens, souvent le nom même du voyagiste est issu du nom d'un transporteur (Vacances par Wardair, Air Canada Touram, C.P. Holidays, Jet Tour Air France Canada, etc...). La relation entre le tourisme et le transport aérien existe; puisque pour "faire du tourisme" il faut "4" $y$ " aller et qu'à plus de 500 kilometres dé distance, l'avion est l'une des alternatives les plus séduisantes et souvent même une solution incontournable.

Tourisme et transport aérien, la relation est dont placée sous le signe de la dépendance du premier envers le second; c'est la dépendance " "géographique'". Également, sous le signe de l'interdépendance economique où l'on voit au cours des années les intervenants (fabricants d'avion, compagnies aćriennes et voyagistes) prendre l'initiative à tour de rôle (voir la contribution de Michel Langlois à ce numéro de Téoros. "Transport aérien et marketing touristique" ).

Notre consommateur dans l'agence de voyages se situe aux termes du processus de distribution de l'industrie du voyage et du tourisme. L'objectif est la destination, il faut y aller pour obtenir les services convoités. L'agent de voyages offre un forfait composé par un voyagiste, dans lequel la composante transport aérien est comprise; de quel type de transport aérien s'agit-il, comment a-t-il influé sur le prix de vente? La présence rassurante en page frontispice d'une brochure du logo d'un grand transporteur cache-t-elle une contribution de ce dernier aux frais d'impression de cette brochure, et peut-être mème une relation encore plus étroite entre les composantes du forfait? Répondre à ces questions c'est décrire l'aspect économique de cette relation transport aérien/tourisme.

"M. Philippes Sureau est directeur du service marketing/ventes de la firme Trafic Voyages Ltée de Montréal. M. Sureau a été le coordonnateur de ce numéro pour les articles portant sur le transport aerien.

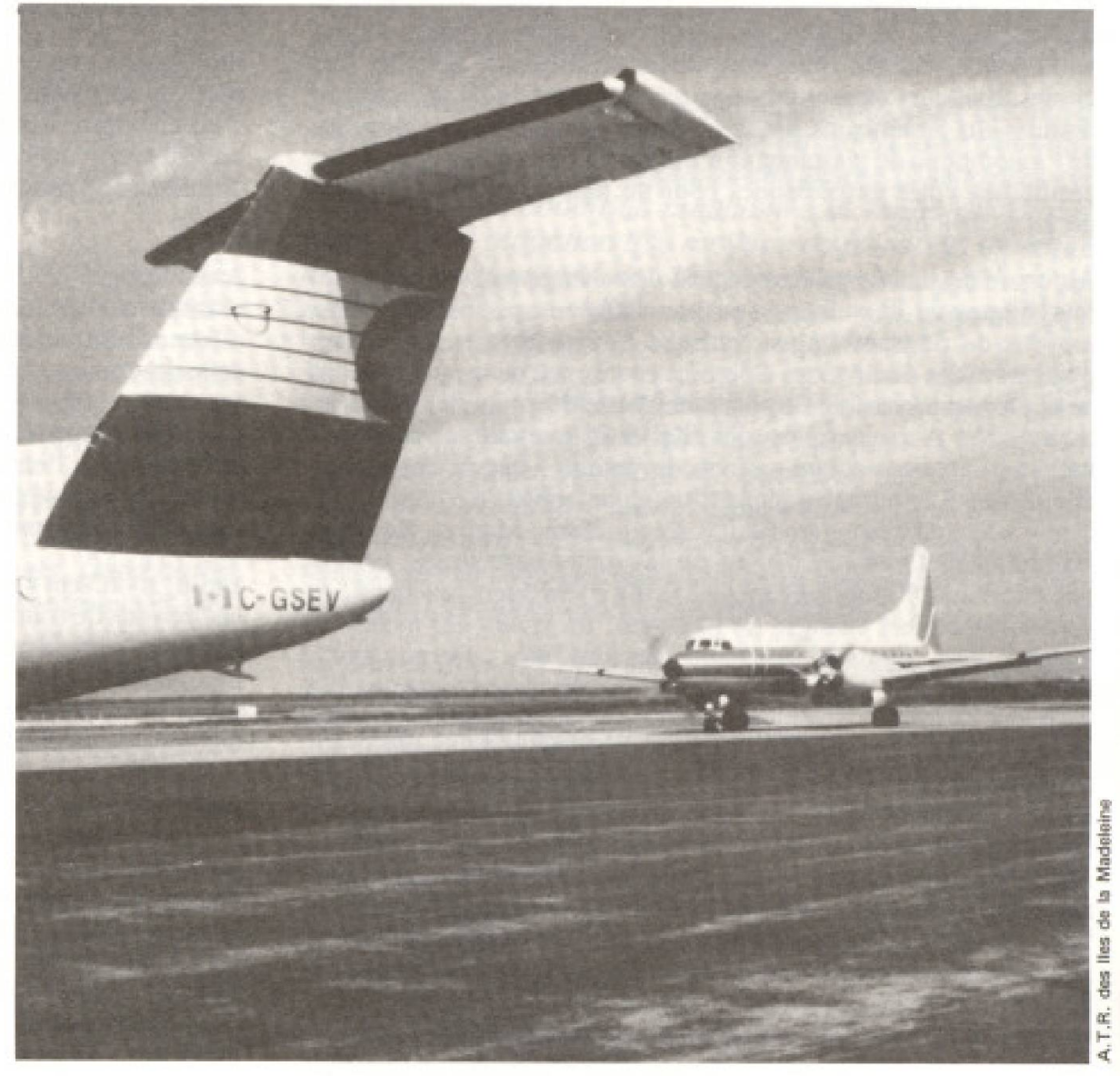

Ce méme consommateur envisage un voyage en République Dominicaine, nouvelle destination vedette au Box Office de 1'hiver québécois; mais depuis quand Puerto Plata fait-il partie des vacances possibles pour la bourse du consommateur? Depuis 1982, année de la construction de l'aéroport international, qui a permis l'atterrissage de jets (type B737 puis DC8 et par la suite gros porteurs) affrétés par des voyagistes, ces derniers mettant en marché des forfaits "République Dominicaine". Dans ce cas précis, la planification de l'opération par Infratur (Office de planification du développement touristique en République Dominicaine), à la suite d'un plan d"intervention F.M.I. (Fond monétaire international); a permis une coordination entre le développement des capacités d'accueil local (le projet Playa Dorada) et l'ouverture de l'aéroport desservant la région. Les infrastructures ont ainsi un impact déterminant sur le développement du tourisme en conjonction avec le transport aérien.

Une bonne façon de mesurer le succes de l'opération dont nous parlons a Playa Dorada consisterait à comparer la fréquentation de l'actoport "La Union" de Puerto Plata et le remplissage des hôtels de Playa Dorada.

Notre consommateur l'a échappé belle! Le voyage qu'il prévoyait a failli lui couter plus cher que son agent de voyage ne lui avait tout d'abord confirmé.

En effet, le tarif aérien Montréal/Paris dans la brochure de tel voyagiste a été tout 
d'abord refusé par les services compétents de l'Aviation civile française, puis suite à de pénibles tractations, ce même tarif a ćté toléré. L'environnement légal dans lequel baigne le transport aérien a des implications majeures pour le développement du tourisme. Cet environnement est en train de changer et toutes les données ne sont pas encore disponibles. Cependant, il faut décrire le cadre juridique de la relation tourisme/transport aérien, en particulier en ce qui a trait aux vols affrétés.

Vols affrétés, vols réguliers, compagnies aériennes à horaires fixes ou compagnies aćriennes offrant des vols à la demande: voila les deux grandes familles du transport aérien des passagers; elles répondent à des besoins différents. Cependant, nous verrons que les différences s'amenuisent; la bonne planification de l'utilisation d'une flotte pouvant amener l'une à s'occuper des affaires de l'autre et vice-versa.

Voici donc quelques aspects des relations de ce couple tourisme et transport aérien: - dimension économique;

- la dépendance géographique;

- l'impact des structures;

- l'environnement légal.

Certains des textes de ce numéro de Téoros traitent plus particulièrement de ces aspects et je voudrais développer sommairement ici certains aspects économiques de cette relation, les relations entre tourisme et vols nolisés ou vols réguliers, et tout d'abord situer le cadre légal dans lequel se déroule "l'action".

\section{L'environnement légal}

Le transport aćrien est une activité liếe à la souveraineté nationale. Les droits de survol, d'atterrissage et de commerce liés au transport des personnes et des biens et de la poste, sont définis dans une convention dont sont signataires tous les pays nembres de O.A.C.I. (Organisation de l'aviation civile internationale) dont le siège est à Montréal.

Cette convention constituée de l'O.A.C.I. est connue sous le nom de Convention de Chicago (signée à Chicago le 7 décembre 1944).

Constatant l'importance du transport aérien comme moyen de communication entre les nations et les peuples, cette convention a pour but de définir certains principes qui garantissent la sécurité des passagers et le développement du transport aérien d'une maniere ordonnée respectant le principe de l'égalité des chances et la santé économique des intervenants.

Ainsi les principes de sécurité, d'égalité des chances et de viabilité économique sont les piliers de cette convention.

\section{Étude d'un cas: Montréal/Paris 1985-1987}

La baisse ininterrompue des tarifs débute en 1984. Air France et Air Canada publient un tarif aller-retour dit super apex (APEX pour Advanced Purchase Excursion Fare) a $898 \$$ (départs au mois de juillet) sur la relation Montréal/Paris. Ces tarifs sont dirigés vers une clientèle exclusivement touristique, de vacanciers et rapprochements familiaux.

Le tour opérateur français, Nouvelles Frontières, s'engouffre dans l'espace laissé libre entre les prix de revient d'un vol affrété (Quebecair) et les prix de vente publics publiés par les transporteurs réguliers. Les tarifs de Nouvelles Frontières (598\$) font recettes et l'opération est un sucoès. Les transporteurs réguliers doivent finalement ajuster leurs prix à la baisse.

L'année suivante, l'offre en vols affrétés est encore plus forte et la situation est la suivante: 14 vols affrétés par semaine sur la relation Montréal/Paris (Nationair 5, Quebecair 5, Wardair 4). Une part du marché grandissante échappe au transporteur régulier (environ $10 \%$ de part de marché en moins pour Air Canada).

À la fin de l'été 1985, deux transporteurs affrétés (Quebecair, Nationair) annoncent leur intention d'opérer des vols toute l'année. Le prix public proposé $398 \$$.

En réaction à cette situation, la Direction générale de l'aviation civile française (D.G.A.C.) informe les transporteurs de son intention de leur retirer leurs droits de transport sur la relation si les tarifs ne sont pas augmentés à un niveau minimum. Ainsi une autorité française s'estime compétente en matière de prix au départ du Canada vers la France. Les arguments de la D.G.A.C. sont fondés sur l'article 5 de la Convention du Chicago, chapitre 11 , concernant les droits des vols affrétés:

"Each contracting State agrees that all aircraft of the other contracting States, being aircraft not engaged in schedule international air services shall have the right, subject to the observance of the terms of this Convention, to make flights into or transit non-stop across its territory and to make stops for non-traffic purposes without the necessity of obtaining prior permission, and subject to the right of the State flown over to require landing. Each contracting State nevertheless reserves the right, for reasons of safety of flight, to require aircraft desiring to proceed over regions which are inacessible or without adequate air navigation facilities to follow prescribed routes, or to obtain special permission for such flights.

Such aircraft, if engaged in the carriage of passengers, cargo, or mail for remuneration of hire on other scheduled international air services, shall also, subject to the provisions of Article 7, have the privilege of taking on or discharging passengers, cargo, or mail, subject to the right of any Siate where such embarkation of discharge takes place to impose such regulations, conditions or limitations as it may consider desirable".

La réponse des transporteurs canadiens porta le débat sur le plan commercial; en effet, les arguments légaux des Français étaient fondés ainsi que nous l'avons mis en évidence en soulignant le texte de la Convention de Chicago.

À l'automne 1986, Québócair a abandonné son secteur des vols nolisés longue portée, et Nationair a acquis les appareils DC8-63 de Quebecair. Nationair se propose d'operer deux vols au cours de l'hiver 1986/87 l'un pour le voyagiste Nouvelles Frontières, l'autre pour le voyagiste Trafic Voyages. Les autorités françaises (D.G.A.C.), nonobstant d'autres faits, demandent au transporteur Nationair de n'assurer qu'un seul vol. Cette fois, les Français s'appuient sur le principe de la réciprocité de pavillon. En effet, aucun transporteur français n'opère de vols affrétés sur la relation Montréal/Paris pendant la période concernée, et s'appuyant sur l'énoncé du préambule de la Convention de Chicago, les Français exigent qu'à chaque "pavillon" canadien soit en opposé un "pavillon" français, voici la partie du texte du préambule concerné:

"Therefore, the undersigned governments having agreed on certain principles and arrangements in order that international civil aviation may be developed in a safe and orderly manner and that international air transport services may be established on the basis of equality of opportunity and operated soundly and economically".

Dans ce dernier cas, les autorités françaises auront également gain de cause et si des arguments commerciaux ont empêché la D.G.A.C. d'exercer un contrôle sur les prix publics (notre premier exemple), dans le cas de la "querelle des pavillons" le Canada a dû accepter la situation et au cours de l'hiver 1986/87 un seul vol affrété a été opéré sous pavillon canadien.

Quelles sont les libertés aériennes? Ce sont les droits que détiennent les transporteurs réguliers d'effectuer un certain nombre de relations aériennes en regard de certains territoires. Ainsi, la première liberté autorise un transporteur à survoler un territoire; la seconde lui permet de s'y poser pour une escale technique; la troisième d'y débarquer des passagers; la quatrième d'y embarquer des passagers à destination d'une troisième destination. Ces autorisations sont le fait d'une multiplicité d'ententes bilatérales puisque la Convention de Chicago, article 6 , donne pleine autorité à chaque état souverain d'accorder ou non le droit d'opérer des vols réguliers à destination de son territoire. "No scheduled international air service
may be operated over or into the territory
of a contracting State, except with the spe- 
cial permission or other authorization of that State, and in accordance with the terms of such permission or authorization".

Nous avons survolé certaines des dimensions de l'espace juridique dans lequel évolue le transport aérien. La Convention de Chicago traite des grands principes ainsi que nous l'avons vu. En ce qui concerne les tarifs réguliers et leurs règlements, les transporteurs réguliers se sont regroupés dans 1'I.A.T.A. dont l'un des mandats principaux est l'amélioration des revenus des transporteurs. Un comitế connu sous le nom de C.Y.I.P. (Canadian Yield Improvement Panè) a même été formé pour tendre vers ce but; sur ce comité siège des représentants des transporteurs réguliers desservant le Canada, sous la présidence d'Air Canada. Chaque pays réglemente, plus ou moins, le transport aérien et les compagnies aćriennes sur son territoire.

Au Canada, le gouvemement fédéral a deux organismes qui interviennent dans ce secteur: le Comité des transports aériens (C.T.A.) de la Commission canadienne des transports (C.T.C.) chargé de l'application des règlements sur le transport aérien (R.T.A.), et le ministère Transport Canada chargé du contrôle des aéronefs et des conditions d'utilisation de ces aéronefs.

La nouvelle politique sur le transport au Canada, connue sous le vocable "Aller sans entrave" "a déjà indiqué l'orientation que le gouvernement fédéral entend donner au transport aérien dans l'avenir immédiat: liberté d'entreprendre au sud de $49^{\circ}$ de latitude et maintien du statu quo réglementé au nord du $49^{\circ}$ de latitude. Ainsi la C.T.C. devrait en grande partie perdre son rôle de gendarme et de régulateur de l'entrée sur le marché des nouveaux transporteurs*.

Le certificat attribué par Transport Canada indique que les operations du transporteur sont effectuées d'une manière conforme aux normes de Transport Canada (agrément de l'organigramme de la Compagnie, de son manuel d'opération et des aéronefs).

\section{Tourisme, vols réguliers, vols nolisés}

L'obtention d'une route régulière était un actif précieux pour un transporteur, au Canada; la C.T.C. a le mandat de donner et éventuellement de suspendre ces autorisations. Le transporteur a alors l'obligation d'assurer, à heure fixe, un service aérien entre les deux points concernés et le privilège de voir ce droit protégé.

Le transporteur à la demande, s'assure de l'affretement complet de l'avion avant d'opérer son wol. Ainsi, il est sûr de la rentabilité de ce vol puisque tout l'avion est réservế par un seul client: l'affréteur. Pour prévenir le risque de voir un transporteur affrété s'implanter sur une base "'régu- lière" sur une toute où opèrent des transporteurs réguliers. Les R.T.A. ont interdit à un transporteur à la demande de s'affréter lui-même, détournant ainsi l'esprit de la loi de l'aéronautique. Cependant, cette façon de distinguer une offre régulière et une offre affretée a perdu son sens avec l'apparition des vols nolise-réguliers. En effet, devant l'importance de la demande touristique, marché de voyagiste, plusieurs d'entre eux affrètent sur une base annuelle certains vols (Nouvelles Frontières et Trafic Voyages Montréal/Paris depuis 1984, Mirabelle Tours Montréal/Puerto Plata depuis 1982), Certains transporteurs à la demande ont même basé leur développement sur leur filiale voyagiste qui affrète les appareils de la maison-mère (Wardair et Vacances par Wardair).

Ainsi la relation vols affrétés, vols exceptionnels et vols a horaire fixe, vols réguliers, tend à s'estomper au profit d'un autre concept, lié au monde de distribution du produit et au déplacement vers le voyagiste du risque et du profit.

Le trafic des gens d'affaires entre deux points donnés d'une relation aérienne a tendance à culminer pendant la semaine. Ainsi le nombre de vols offerts entre Montréal/Toronto est beaucoup plus important du lundi matin au vendredi soir que le samedi et le dimanche.

Le gestionnaire de la flotte d'une compagnie aćrienne régulière pourra juger opportun d'offrí a la location ses appareils qui restent à terre pendant la fin de semaine. De plus, le marché des vacances est plus concentré en fin de semaine renforçant ainsi cette tendance. Voici donc l'apparition de compagnies mixtes régulières en semaine sur un réseau, affrétées en fin de semaine sur des destinations vacances (ex: P.W.A. C.P. Air, Quebecair, Nordair).

À l'opposé, un transporteur à la demande peut, considerant que sa flotte est à terre en semaine pour cause d'inactivité chronique dans le secteur vacances (ses coüts fixes sont couverts par ses opérations de fin de semaine) alors tenter de mettre une opération régulière sur pied à coût modique (vols de Nationair sur Bruxelles à partir du mois de mai 87). L'autre motivation majeure étant l'acès au réseau de distribution informatisć du type de Reservec (Air Canada) et au système de règlement bancaire intégré connu sous le label B.S.P. (Bank Settlement Plant). Wardair a ainsi choisi cette voie pour plusieurs de ses opérations auparavant nolisées (route domestique).

Les transporteuts choisissent ainsi le mode de distribution qui convient le mieux à la commercialisation de leurs produits: vols réguliers haut de gamme pour des vols à vocation de service affaire, vols affrétés service réduit, bas tarif, pour des vols à vocation touristique. Sur certaines destinations, le même transporteur peut ainsi offrir des vols réguliers et des vols affrétés. Il ne s'agit plus ici de gérer une demande accrue exceptionnelle, mais plutôt d'offrir au consommateur un produit different en termes de tarif et d'image.

Les transporteurs ont envahi le domaine de la distribution et de la "fabrication" de produit touristique et nous verrons sûrement, comme déjà dans le cas en Europe (Conair en Scandinavie, par exemple), des voyagistes "envahir" le domaine aérien.

Le consommateur, que nous évoquions au début de cet article, en ramassant ces brochures de voyages constellées de logo de transporteurs est à l'origine de ce processus. Le passager touristique a le choix de partir ou de ne pas partir, d'aller à telle destination ou à telle autre. Les transporteurs ainsi que l'a décrit Michel Langlois, (voir note plus haut) ont dû s'intếresser à ce passager au choix discrétionnaire, qui doit être séduit par les prix de plus en plus compétitifs pour assurer les taux de remplissage nécessaires pour survivre dans de nouvelles conditions économiques. La participation du transporteur a pris la forme d'une commission de plus en plus forte consentie au "forfaitiste", ou de prix net de plus en plus bas. Il faut offrir un forfait au meilleur prix pour s'assurer une part de ce marché touristique. L'avantage de cette façon de faire réside dans le fait que le coût du transport aérien est noyé dans une prestation plus complète, ainsi il n'entre pas en compétition avec les tarifs "avionseulement".

Cette technique poussée à l'extrême a mené certains voyagistes, sous la houlette des transporteurs, à réduire la part terrestre au stricte minimum et ainsi utiliser l'aérien pour "subventionner" des prestations à terre dont le prix apparent pour le public consommateur a perdu tout contact avec la réalitế. Ainsi on offre des nuits d'hôtels de qualité pour quelques dollars. Les transporteurs ont vite perçu que pour atteindre le public des touristes voyageurs, il fallait habiller le produit avion et devenir voyagiste.

Le tourisme est devenu essentiel aux compagnies aériennes, leur avenir, leur rentabilité, leur croissance sont bâtis sur cé secteur. Les transports liés aux voyages d'affaires n'assureront pas seuls ce développement et le sort de ces deux domaines sont définitivement associés pour longtemps. $f$

- Les transporteurs aériens doivent obtenir un permi et un certificat, Le permis est donné par la C.T.C. II indique ou moyen de chiffres le type d'opération que le detenteur peut effectuer et par une lettre le tyce d'appareil qu'il peut utiliser pour ce faire. Le type d'appareil est limité par su masse maximale au decollage lex: Le 9-4 H est un permis d'operer des wols $A$ partir d'un point au Canada au sud du $49^{\circ}$ â destingtion de n'importe qual point dans le monde avec des appareils dont la masse maximale au décollage est de 325000 lbs, ou plus, c'est-a-dire des gros porteurs de type B747, DC10 ou L1011). 